\title{
Las emprendedoras en el medio rural. El caso de estudio del Somontano aragonés
}

\author{
Cecilia Serrano Martínez \\ Universidad de La Rioja \\ cecilia.serrano@unirioja.es \\ Diana Valero Errazu \\ Universidad Antonio Nebrija \\ diavalero@gmail.com \\ Carlos Gómez Babillo \\ Universidad de Zaragoza \\ carlosgomezbahillo@gmail.com
}

Resumen: Lo rural se ha asociado a una forma de «hábitat» que implicaba la actividad económica agraria, que constituía el centro de la vida familiar y vinculaba a sus componentes. En las últimas décadas, estos «hábitats rurales» se han ido transformando $y$ ha acontecido un aumento del autoempleo femenino como una alternativa de incorporación al mercado laboral. El objetivo del artículo es conocer las posibilidades y experiencias laborales de las mujeres de la zona rural del Somontano (Aragón, España), a través de catorce entrevistas realizadas a mujeres empresarias. Los aspectos más destacados son la experiencia como herramienta profesional, incertidumbres, conciliación y las ayudas recibidas en su emprendimiento.

Palabras clave: mujer; empresaria; medio rural; autoempleo. 
Woman and enterprise in rural environment. Entrepreneurial women in the Aragonese Somontano

Abstract: The concept of "rural" has been associated with a form of "habitat" which involved an agrarian economy and was the center of family life. In recent decades these "rural habitats" have been transformed and female self-employment has increased as an alternative to entering the labor market. This paper aims to identify the possibilities and work experiences of women in the Somontano region (Aragon, Spain), through fourteen interviews with women entrepreneurs. The most notable elements are experience as a professional tool, uncertainties, work/life balance, and the aid they received during their entrepreneurship.

Key words: women; business woman; rural environment; habitat; self-employment. 


\section{Planteamiento del problema}

\subsection{La contextualización. El entono rural hoy}

La penetración de los medios de comunicación social en el ámbito personal y familiar, las nuevas funciones que el medio rural tiene como lugar de ubicación de actividades industriales y como espacio de expansión y de ocio, la movilidad de su población, el crecimiento de los intercambios comerciales y la posibilidad de acceso a redes de información e intercambio han modificado las costumbres, expectativas y aspiraciones de las gentes del campo, y, de una manera especial, las de las mujeres que participan cada vez con más frecuencia en redes sociales (Vega y Buzón, 2016). El hábitat rural ha adquirido características urbanas a causa de la necesidad de nuevos espacios que requiere la actividad industrial y comercial y un sector de servicios en pleno proceso de expansión. Antiguas zonas rurales, bien situadas y con buenas infraestructuras de comunicación y transporte, han desarrollado un importante sector secundario y de servicios. A ello hay que añadir el crecimiento de urbanizaciones, con características típicamente urbanas, que se han convertido en lugar de residencia definitiva, ocasional de temporada o de fines de semana para los residentes. Frente a esta nueva realidad existe también otra España rural que languidece por el despoblamiento, envejecimiento de su población, sin alternativa económica a la actividad agraria de subsistencia, carencia de infraestructuras, alejada de vías importantes de comunicación, falta de servicios básicos, etc. (Pinilla y Sáez, 2017; Jurado y Pazos, 2016).

En España, como sucede en el resto de Europa, algunas zonas rurales han experimentado una importante transformación como consecuencia de las nuevas funciones que tiene el medio rural para los residentes de la ciudad. Este entorno tiene un valor en sí mismo y sus recursos naturales son valorados positivamente por la población residente en las ciudades, que ha descubierto en la naturaleza un lugar ideal para el descanso y desarrollo de actividades de ocio y tiempo libre. Esto ha repercutido en las relaciones familiares y sociales de sus residentes, en las expectativas de sus habitantes y en la fisionomía de algunos pueblos.

En las últimas décadas, la expansión de la agricultura orientada hacia la calidad y otras actividades vinculadas al turismo, a la valorización del patrimonio y paisaje, a la utilización de energías renovables y al desarrollo de actividades relacionadas con las tecnologías de la información y comunicación están permitiendo aprovechar el potencial y las posibilidades de las zonas rurales. En el desarrollo de estas actividades tienen un papel importante las mujeres, ya sea como titulares de pequeñas empresas o a través de la prestación de servicios como autónomas. «La transformación económica ha coincidido en el tiempo con la creciente in- 
corporación de las mujeres al mercado laboral, en lo que han confluido varias razones: el trabajo remunerado es para buena parte de las mujeres una vía de autonomía y realización personal; a ello se suman las necesidades de las familias rurales de diversificar sus ingresos y una oferta creciente de puestos de trabajo en el sector terciario» (Ministerio de Medio Ambiente y Medio Rural y Marino, 2011: 246-247). Por ello, su contribución al desarrollo endógeno es una realidad, de manera que «su presencia y actividad en el territorio, no solo económica sino también social y cultural, está dibujando una nueva ruralidad acorde con las necesidades del siglo xxı» (Hervás et alii, 2018: 723).

\subsection{Posibilidades laborales de la mujer rural}

La presencia de la mujer en la actividad productiva es crucial para la supervivencia y sostenibilidad del medio rural, por lo que es necesario generar expectativas y posibilidades reales para nuevos asentamientos (Díaz, 2005). El papel de la mujer es determinante no solo para el desarrollo económico sino muy especialmente para la transformación social y cultural y «el emprendimiento rural en los últimos años ha adquirido un papel cada vez más protagonista, ya que contribuye a la modernización y sostenibilidad de estos entornos gracias a la creación de nuevas empresas que, en muchas ocasiones, implementan nuevos modelos de negocio distintos de los tradicionales» (Esteban, Pérez y Gargallo, 2018: 1). Las mujeres están contribuyendo al desarrollo del cooperativismo, a frenar el éxodo rural y favorecer la sostenibilidad de las zonas más deprimidas y deshabitadas (Esteban et alii, 2018). La igualdad de género en las oportunidades laborales es «un objetivo prioritario en el ámbito rural, que provocará desarrollo y contribuirá a la mejora de la sostenibilidad en este entorno» (Cabezas, 2018: 62).

Esta preocupación y toma de conciencia de las mujeres rurales sobre la necesidad de tomar la iniciativa y asumir un rol activo en la actividad económica se refleja en el apoyo que ha tenido el proyecto Red Europea de Mujeres Empresarias del Medio Rural, cuyo objetivo ha sido crear una red de empresarias de pymes en el ámbito rural, a nivel nacional e internacional, para construir un entorno de mujeres empresarias y/o emprendedoras.

Como señala García Sanz (2004b: 109), «el grupo que está llamado a crecer es el de las autónomas y el de las empresarias, a estancarse el trabajo en cooperativas, y a disminuir las ayudas familiares. Es un proceso de racionalización de la ocupación que sin duda alguna está afectando a la mujer rural». Las mujeres toman la decisión de realizar una actividad independiente como autónomas, por independencia económica, por ser el soporte de la familia en los momentos de crisis de empleo del varón, por necesidades personales (Olson y Walker, 2004: 
244-251), o por la falta de oportunidades y no tener la posibilidad de conseguir un empleo asalariado, en los nichos laborales que tradicionalmente se han reservado para ella (Bruin et alii, 2006: 585-593).

El aumento del autoempleo femenino es una alternativa cada vez más extendida entre las mujeres que se incorporen al mercado laboral (Buttner, 2001), y el número de empresas gestionadas por ellas es uno de los apoyos fundamentales de las políticas de desarrollo endógeno rural. El medio ofrece, en ocasiones, posibilidades y oportunidades de negocio para mujeres emprendedoras, sin que tengan que desplazarse para ello a la ciudad o a las zonas urbanas. «Un nuevo elemento a considerar dentro de los que inciden positivamente en las emprendedoras es la toma de conciencia por su parte acerca de las posibilidades que se abren en los medios rurales, en relación a las nuevas y crecientes demandas que a menudo no encuentran respuesta adecuada en la oferta, o a la explotación de recursos locales hasta entonces desaprovechados» (Nieto, 2003: 265). El trabajo por cuenta propia es una alternativa cada vez más extendida entre las mujeres rurales, ya que permite su incorporación a la actividad laboral, ante la crisis progresiva de empleo asalariado.

Las mujeres rurales realizan su actividad «en cuidados personales, donde son mayoría; pero también en los sectores de comercio y hostelería. En el resto de servicios, donde se incluyen mayoritariamente los servicios a empresas, la tasa de feminidad es mínima» (Escobar et alii, 2005: 79). En industria su presencia es más reducida y aparecen en empresas agroalimentarias y textiles debido a su menor coste. Como señala García Sanz (2004b: 101), «la importancia del sector industrial en el mundo rural ha tenido dos caminos de penetración, uno el de la industria agroalimentaria, que ha dado una opción también de trabajo a las mujeres rurales, $y$ otro, el textil, que ha buscado en la mano de obra femenina una fórmula para disponer de un trabajo seguro y barato». Existen nichos de actividad en los que la mujer tiene mayores posibilidades, sobre todo en el mundo de la hostelería y de la restauración, donde se encuentran las mujeres más dinámicas y emprendedoras, con un extraordinario empuje empresarial y con un acreditado dinamismo económico.

Las oportunidades que la mujer tiene para desarrollar una actividad remunerada están condicionadas y determinadas por los cambios que se producen en el entorno rural, como consecuencia de la terciarización de las actividades económicas. Según el estudio realizado por Langreo y Benito (2007), resulta que el $80 \%$ de los proyectos están relacionados con el patrimonio y turismo, a los que se dedica la mayor parte de las inversiones, así como a servicios a la población.

No obstante, si cualquier iniciativa empresarial en el medio rural tiene mayores dificultades, en el caso de «las mujeres se sacan adelante supliendo la falta de 
capital con trabajo, sopesando muy bien los riesgos (yendo "muy poco a poco") y apoyándose en el valor, no estrictamente económico, que se otorga a la creación de la empresa o negocio, como signo de afirmación e independencia personal» (Sampedro y Camarero, 2007: 121-143). Las mujeres reconocen que llegar hasta aquí ha sido más difícil de lo esperado, aunque ha merecido la pena. A los problemas inherentes de la profesión se han añadido otros, simplemente por el hecho de ser mujeres y haber asumido la responsabilidad de una empresa (García, 2004a: 112).

El perfil medio de las mujeres empresarias en el medio rural español se caracteriza por (Consejo Superior de Cámaras de Comercio. Ministerio de Agricultura, Alimentación, y Medio Ambiente, 2012: 107-108):

- La edad media de la mujer emprendedora en el medio rural es de 43,21 años, seis años superior a la media nacional de las mujeres emprendedoras.

- El 55\% de las mujeres empresarias rurales han realizado un trabajo previo como asalariadas, y únicamente el $28 \%$ de estas mujeres se inician en el mercado laboral por la vía del autoempleo.

- La mujer empresaria rural tiene un nivel cualificación superior (60\%), lo que contrasta con la media general, en donde prevalecen los estudios secundarios. Por el contrario, la formación específica en materia de creación de empresas es menor entre las mujeres rurales.

La cuestión de la financiación suele ser el principal problema para que se consoliden las iniciativas laborales no asalariadas por parte de las mujeres, especialmente en el medio rural: «Las diferencias entre los comienzos y los momentos posteriores radican en que al inicio, el principal inconveniente está relacionado con la falta de fondos, mientras que en la continuación, el mayor problema señalado es la competencia» (Escobar, Filardo y Ferrer, 2005: 159). En otras ocasiones, a ello se añade la falta de experiencia en la actividad y el desconocimiento de la gestión financiera, a pesar del buen nivel formativo que poseen. No obstante, en el medio rural, son más relevantes los factores informales como la percepción de habilidades para emprender, las redes sociales y el rol familiar, que los formales como la financiación, políticas de apoyo no económicas y formación (Álvarez et alii, 2012).

A ello hay que añadir las limitaciones con las que se encuentra la mujer para realizar una actividad económica de manera autónoma e independiente, dado que deben «hacer un esfuerzo extra, en comparación con sus homólogos masculinos. Por otro lado, las responsabilidades familiares siguen condicionando la actividad empresarial de muchas mujeres y esto se refleja en una menor dedicación horaria, 
en la limitación del crecimiento de la empresa y, sobre todo, en su dificultad para participar en cursos de formación o en redes de negocio fuera de su horario de trabajo» (Jiménez, 2011:158).

Pero desde hace un par de décadas cada vez es mayor el protagonismo de la mujer emprendedora en el Somontano de Barbastro, y como señalaba Santiso (2002: 175), especialmente «en la reestructuración de las zonas rurales [...], siendo parte activa y titulares de otras actividades diferentes a las tradicionales en estos municipios, relacionadas con la creación de pequeñas empresas de servicios, en lo relacionado con la manufacturación de productos locales artesanos $y$ alimentarios, y sobre todo con el turismo rural, que es la fuerte apuesta con un potencial dinamizador de estas zonas».

\section{Diseño metodológico}

La mayoría de las mujeres emprendedoras en el mundo rural lo hacen por herencia directa o por matrimonio, habiendo participado de los negocios desde una temprana edad (Sampedro y Camarero, 2007). El objetivo de este estudio es dar voz a lo que se ha considerado una minoría, aquellas mujeres que han decidido ser emprendedoras desde cero y conocer las posibilidades y experiencias laborales de éxito de estas mujeres con el fin de poder favorecer políticas de autoempleo que no se basen en la herencia familiar o el matrimonio. Para ello, como se ha hecho en otros estudios (Cruces y Palenzuela, 2006; Sánchez-Flores, Royo, Lacomba, Marín y Benlloch, 2014; Sampedro y Camarero, 2007), se ha realizado un estudio cualitativo incorporando las voces de 18 mujeres empresarias de la zona a través de entrevistas semiestructuradas. En total han participado $18 \mathrm{mu}$ jeres, ya que, en algunos casos, la entrevista era realizada simultáneamente a dos socias de un mismo negocio.

La mayoría de estas informantes desarrollan su actividad en el sector servicios (comercio, restaurantes y hospedajes, servicios sociales y actividades sanitarias). De las 14 entrevistas, 10 se dedican al sector servicios, 3 al sector industrial y 1 al sector de construcción. En cuanto a la edad, han participado 8 mujeres mayores de 40 años y 6 menores, lo cual nos aporta un abanico más contrastado de trayectorias laborales e incluso vitales. La diferenciación por edad (mayor o menor de 40 años) persigue el objetivo de conocer si existen diferencias generacionales en su discurso; y en función del sector en el que ejercen su actividad, con el objetivo de conocer diferentes realidades empresariales, en función de la dedicación a una $\mathrm{u}$ otra actividad.

Los perfiles entrevistados son los siguientes: 
- Entrevista 1 (E1). Tienda multiservicio. Sector servicios. (Mujer, $<40$ años).

- Entrevista 2 (E2). Repostería artesana. Sector industrial. (Dos mujeres, $>40$ años).

- Entrevista 3 (E3). Mermeladas. Sector industrial. (Dos mujeres, < 40 años).

- Entrevista 4 (E4). Peluquera. Sector servicios. (Mujer, $<40$ años).

- Entrevista 5 (E5). Restaurante y posada. Sector servicios. (Mujer, > 40 años).

- Entrevista 6 (E6). Agencia de viajes. Sector servicios. (Mujer, $<40$ años)

- Entrevista 7 (E7). Bordados industriales. Sector industrial. (Mujer, $>40$ años).

- Entrevista 8 (E8). Actividades para mayores. Sector servicios. (Dos mujeres, $>40$ años).

+ Entrevista 9 (E9). Taller de cerámica. Sector servicios. (Mujer, < 40 años).

- Entrevista 10 (E10). Rehabilitación de edificios. Sector construcción. (Mujer, $<40$ años).

- Entrevista 11 (E11). Residencia y ayuda a domicilio. Sector servicios. (Dos mujeres, < 40 años).

- Entrevista 12 (E12). Restaurante y apartahotel. Sector servicios. (Dos mujeres, $>40$ años).

- Entrevista 13 (E13). Servicios de interpretación del patrimonio. Sector servicios. (Mujer, $>40$ años).

- Entrevista 14 (E14). Aula de pintura itinerante. Sector servicios. (Mujer, $>40$ años).

El diseño de la entrevista está formado por cuatro preguntas abiertas, a las cuales se han incorporado cuestiones concretas, en función de las respuestas recibidas. El guion utilizado fue el siguiente:

- ¿Cómo fue el comienzo, desde la idea inicial del proyecto emprendedor hasta su desarrollo definitivo?

- ¿Cuáles fueron las dificultades iniciales?

- Aspectos relevantes vivenciados a lo largo de todo el proceso.

- Resultados alcanzados más destacables. 
Los discursos obtenidos han sido transcritos y analizados a través de la herramienta informática de análisis cualitativo HyperResearch. Tras la transcripción se ha llevado a cabo un análisis siguiendo la metodología comunicativa, lo que nos ha permitido analizar los elementos transformadores (aquellos que permiten superar las dificultades) y los exclusores (aquellos que han supuesto barreras) (Flecha, Vargas y Dávila, 2014). La utilización de esta metodología de análisis resulta especialmente interesante ya que permite identificar las fortalezas en las que deben basarse las políticas, así como las barreras que deben tratar de superar.

El contexto geográfico de la investigación, la comarca del Somontano (Aragón), se caracteriza por una actividad económica favorecida por su estratégica ubicación geográfica, dentro del eje socioeconómico Aragón-Cataluña y Navarra-País Vasco. La economía comarcal es fundamentalmente agraria, aunque su pérdida de importancia relativa es un hecho en algunos municipios, en los que predominan actividades industriales y comerciales distintas de las del sector primario. Se trata de pequeñas empresas, muchas de ellas subsidiarias del tercer terciario, en pleno proceso de crecimiento y expansión. Barbastro es la capital administrativa, educativa, comercial e industrial de una amplia área de influencia que rebasa el Somontano. En esta ciudad se produce la mayor parte de la producción, especialmente en los sectores secundario y de servicios, concentrándose en ella las principales fuentes productivas, y produciéndose más de la mitad de la renta que se genera en el espacio comarcal.

\section{Resultados y discusión}

En los últimos treinta años se ha incrementado el nivel educativo y laboral de las mujeres. No obstante, el mercado laboral no cubre todas las demandas laborales que se producen, y el índice de desempleo femenino continúa siendo una cuestión por resolver. Por ello, el autoempleo ha pasado a convertirse es una verdadera oportunidad para las mujeres del Somontano de Barbastro, como alternativa especialmente en las situaciones de crisis económica en las que las posibilidades de empleo para la mujer se ven reducidas, y como opción de vida para asentar la población del medio rural (Fábregas, 2010).

Se presentan a continuación los resultados divididos en tres secciones, la primera dedicada a los inicios del negocio; la segunda, sobre el proceso de consolidación, y la tercera, la influencia en la vida personal y familiar del proceso de emprendimiento. En los tres casos se señalan los elementos tanto inclusores como exclusores detectados. 


\subsection{Motivación e iniciativa}

Los tipos de negocios que aparecen se encuentran principalmente dentro del sector servicios, destacando restaurantes, hospedajes, servicios sociales y actividades sanitarias. Todas las emprendedoras de este estudio comienzan sus negocios de cero, utilizando como motivación sus intereses y habilidades personales, la observación del medio en el que viven y la posibilidad de inclusión de su idea en su medio habitado. En muchas ocasiones, las mujeres se encuentran más motivadas que los hombres para dirigir sus propias empresas, dadas las barreras que perciben en el mercado para aprovechar su potencial humano y profesional como asalariadas (Heilman y Chen, 2003: 347-365).

El primer elemento transformador detectado ha sido la motivación de permanencia en el lugar de residencia como elemento fundamental a la hora de emprender. Lo que implica que las mujeres son un agente sustancial a la hora de fijar empleo y evitar la despoblación rural. Todas las entrevistadas han decidido desarrollarse profesionalmente en la zona en la que deseaban vivir, en su medio rural. Este es el caso de la peluquera que decide montar su negocio en su pueblo por su deseo de continuar viviendo en este. La profesional de bordados textiles desea estar cerca de su familia y revitalizar el pueblo, y estos son los impulsos fundamentales que la llevan a crear su empresa. También destacan que el emprendimiento rural permite desarrollarse con base en sus aficiones y gustos, y crean su propia empresa basándose en ellos. Esto supone un elemento que puede resultar atractivo para atraer a otras mujeres emprendedoras a zonas rurales.

Siempre nos habían gustado estas cosas de la cocina, ya vendíamos alguna mermelada, sin tener así creado el obrador. Entonces nos decidimos. (E3)

Acabé de rehabilitar esa casa, vi que había cosas que me gustaban, y mucho, y que de ahí podía comenzar una idea de negocio y montarme una empresa. (E10)

Es coincidente la habilidad para encontrar una necesidad no cubierta en su medio, y la rapidez para darle respuesta profesionalmente. Aparecen varios casos en los que realizaron un sondeo sobre las posibilidades del mercado de manera informal, es decir, por medio de la observación directa y el contacto continuado en su entorno, y llegaron a la conclusión de que había nichos por cubrir y decidieron darles respuesta por sí mismas. En más de una ocasión señalan que están realizando un negocio o un servicio novedoso, puesto que nadie lo estaba haciendo en ese momento en su localidad. Este aspecto lo sitúan como ventajoso estratégicamente y como valioso económicamente. Por otro lado, el mejorar un servicio ya existente o buscar la distinción en la marca o en el proceso es otro de 
los criterios tenidos en cuenta, como en el caso de la empresaria de bordados o las empresarias dedicadas a la alimentación.

Yo veía que aquí había muchas ofertas de tiempo libre, y en cambio en los pueblos había pocas ofertas. Para ofrecer a los niños actividades, las madres tenían dificultad para traerlos aquí o para programar cosas por medio de recursos que tuvieran en sus localidades. (E14)

En muchas de las ocasiones observan una serie de necesidades sin cubrir que se centran en aspectos referidos al funcionamiento diario familiar y necesidades básicas, como la alimentación, el cuidado de los mayores y la crianza de sus hijos y de los hijos de los vecinos de sus pueblos. No obstante, están apareciendo diferentes situaciones que nos llevarían a hablar de «nuevas necesidades», como las referidas al turismo rural, servicios de estética y de atención al paciente a domicilio, entre otros.

Se me ocurrió la idea porque aquí no había ningún servicio. Al quitar la peluquería, vi que era una oportunidad para este pueblo y para los que están alrededor, ya que no hay ningún otro servicio de este tipo. (E4)

Porque llegaban los pacientes, necesitaban una residencia ya, y no teníamos esa plaza. Nuestra idea fue intentar llevar la residencia al domicilio. (E11)

La observación directa del entorno es lo que les ha llevado a ampliar sus posibilidades empresariales y a atreverse a innovar con servicios que, años atrás, no era habitual encontrarlos en el medio no urbano, como una agencia de viajes dedicada al enoturismo. El turismo rural ha sido una fuente de inspiración fundamental en varios casos, como la agencia citada y las dueñas del restaurante apartahotel.

Creamos la agencia de viajes porque consideramos que era un servicio que no existe, y que a todas aquellas personas que vienen de centros urbanos el hecho de que hubiese una empresa de servicios le podría facilitar, una empresa como la nuestra. (E6)

La identificación con la actividad que realizan es fundamental en la mayor parte de las entrevistadas, e incluso aparecen casos en los que su idea surge por un hobby, como en el caso de la artesana de cerámica. Existen otros casos en los que las mujeres querían crear su propio negocio, pero no tenían claro a qué dedicarse. En estos casos, indican que lograron perfilar sus intereses con la ayuda y el consejo externo, como el que encontraron en cursos previos a los que asistieron, tal y como indica la rehabilitadora de viviendas. 
En este sentido, es importante señalar que, aunque exista ese interés previo, hay una demanda importante de formación en emprendimiento para que estos negocios tengan éxito, lo que abre un espacio interesante de atención por parte de las Administraciones y que sería interesante tener en cuenta en las diferentes políticas para el medio rural.

En los inicios de instauración de sus proyectos, así como en la actualidad abordada en el siguiente punto, el entusiasmo emprendedor se encuentra tintado de ciertos miedos y complicaciones que hemos considerado elementos exclusores. En cuanto a los inicios, las dificultades que citan tienen que ver con problemas técnicos o aplicados, como en el caso de las dueñas del restaurante y apartahotel, las cuales se enfrentan al problema de la falta de alcantarillado en la parte baja de la casa, en la cual querían construir un pequeño restaurante. Uno de los temas más complicados a los que se enfrentaron fue el de conseguir financiación para poner en marcha su negocio. Como las inversiones necesarias en los inicios son más elevadas, realizaron estudios de viabilidad e intentaron buscar fondos en diversas instituciones, con ayudas públicas y/o con ayuda de la familia. En más de una ocasión, señalan una cierta incomprensión y falta de apoyo a su proyecto por parte de ciertas entidades financieras. Entre estos discursos, aparecen con mayor fuerza los de la dueña del restaurante y posada, y la joven dedicada a rehabilitar viviendas.

Iba con un proyecto debajo del brazo y me presentaba en alguna entidad y se sonreían y decían: «Esta mujer dónde va, ¿no?». (E5)

Una obra vale mucho dinero y yo no lo tenía, entonces, pues vas a un banco, vas a otro, y tú quién eres, pues soy una mujer, y a qué te vas a dedicar, pues voy a hacer esto. Los bancos te miran así y tampoco eres el tipo de persona a la que los bancos le dan dinero fácilmente. (E10)

\subsection{Proceso de asentamiento del negocio}

Una complicación diaria en alguna de las empresarias es la de tener un sueldo fijo a final de mes. Esto está motivado por su situación profesional autónoma, por la cual dependen de los ingresos mensuales para llegar a un sueldo u otro, e incluso para tener un sueldo mensual, el cual en todo caso suele ser variable. Sus ingresos varían dependiendo de las actividades mensuales que tengan o de los clientes que reciban. Del mismo modo, en aquellas que se dedican a la distribución de un producto, como en el caso de la empresa de mermeladas, lo más complicado es encontrar compradores fuera de su medio rural, es decir, ampliar mercado para alcanzar una distribución mayor de su producto. Otro caso similar es el de la 
repostería artesana, ya que indican que el cliente no distingue entre un negocio de grandes dimensiones y uno pequeño como el suyo. Estas empresarias llegan a esta conclusión porque, en definitiva, les exigen la misma producción a las distribuidoras de menor tamaño que a las multinacionales.

Entre los miedos, en ocasiones no se han sentido capacitadas para llevar a cabo su trabajo, como en el caso de la empresa de bordados. Otro miedo es el hecho de que los clientes a los que se dirigen no estén concienciados o no entiendan el servicio que ellas les ofrecen, como le ocurre a la empresa dedicada a realizar actividades para personas mayores, donde observan que «la gente no está acostumbrada a pagar por servicios de prevención». (E8). Algunos de estos pequeños inconvenientes o miedos los terminan solucionando por la experiencia que van adquiriendo con el paso de los años.

Todos estos elementos exclusores coinciden con el punto anterior ya que se basan en un desconocimiento del proceso emprendedor y en falta de conocimientos técnicos, lo que puede ser subsanable con un adecuado programa de formación y acompañamiento. Lo que se constata, en la voz de las entrevistadas, es que la experiencia es la principal herramienta que utilizan en su desarrollo profesional, así como el aspecto que más valoran como clave de su éxito. Cada una de las empresarias nos indica las diversas etapas que les han llevado a alcanzar la experiencia en su trabajo. Las dos maneras más reiteradas en los discursos sobre los modos de alcanzar la experiencia son por medio de un conocimiento previo (realización de cursos, estudios, afición, etc.) y por medio de la puesta en marcha del negocio. En este segundo caso, el modo de adquirir la experiencia fue básicamente en el terreno de trabajo, y por la consiguiente necesidad de especializarse en el tema que iban a desarrollar. En primer lugar, aparecen aquellas que tenían conocimientos previos sobre las actividades que desarrollan, como los de casos de la peluquera, la tejedora, la artesana de cerámica, las hosteleras y las reposteras, entre otras.

En segundo lugar, aparecen las mujeres que no tenían experiencia previa, pero que se volcaron en el aprendizaje de las técnicas que necesitaron para llevar a cabo sus actividades profesionales. No obstante, en todos casos, realizaron cursos para saber llevar la gestión de su nueva empresa. Esta formación les ayudó a tener un aprendizaje previo y les proporcionó una cierta experiencia básica de partida. Los cursos sobre gestión se traducen en un mayor bagaje previo y les aportan confianza en sí mismas y un empoderamiento como gestoras de sus propias empresas. En más de una ocasión, señalan que la experiencia les da «más seguridad». Por eso, la formación es un recurso fundamental, que las empodera como profesionales y les permite superar barreras de género y de clase (Porto et alii, 2015). 
Junto con la valoración concedida a la experiencia, aparecen otras valoraciones personales referidas al conjunto de sus sensaciones y opiniones. Entre ellas, destaca la importancia concedida al «salir de la rutina», que les suponía estar todo el día en casa. Para ellas es positivo sentirse valoradas profesionalmente fuera del hogar. Del mismo modo, reconocen que han tenido que pasar una media de tres a cuatro años para valorar el esfuerzo y comenzar a ver los frutos de su trabajo. A partir de este momento, dicen sentirse valoradas y reconocidas plenamente por su comunidad y por los clientes a los que se dirigen.

Del mismo modo, en el reconocimiento de los apoyos que tienen y han tenido también le dan valor a una serie de ayudas económicas y formacionales externas, que han surgido por parte de programas comarcales y provinciales. Se refieren a subvenciones que han recibido por ser mujeres emprendedoras, así como a la asistencia a cursos de formación financiados. Muchas de estas subvenciones tienen como criterios valorativos la aportación profesional de estas mujeres, ya que están generando un desarrollo rural y evitan la desterritorialización de estos núcleos.

Eso fue el motivo por el que me dieron la subvención, porque yo acercaba al medio rural y facilitaba a las madres de familia esta conciliación de sus hijos. (E14)

En definitiva, se trata de cursos a los que asistieron para ampliar sus conocimientos y para conocer diferentes estrategias de mercado, así como ideas de marketing y otros saberes relacionados con el manejo empresarial aplicado. Estas ayudas y subvenciones son apoyos públicos y proceden de fundaciones o instituciones regionales y comarcales específicas. Entre las funciones de estas ayudas económicas aparecen las de apoyar al comercio, fomentar el desarrollo de las áreas rurales y empoderar a la empresaria rural.

\subsection{Efectos del emprendimiento a nivel personal}

Finalmente, emprender tiene unas implicaciones no solo económicas sino también personales. Entre la experiencia previa y la práctica alcanzada tras el tiempo invertido es coincidente el interés que muestran en seguir avanzando y mejorando las técnicas. Las empresarias rurales se caracterizan por su proactividad y entusiasmo. Nos referimos a diferentes actitudes vitales que aparecen en todos los casos entrevistados. Del mismo modo, se esfuerzan por ofrecer un servicio de calidad y dar respuesta a las necesidades de sus clientes, utilizando sus propias herramientas. Este es el caso de la tienda multiservicio, en la que la dueña habla varios idiomas y ofrece una atención plurilingüe a los clientes para facilitar la comunicación con aquellos habitantes cuya lengua materna no sea la del lugar de origen. 
Sin embargo, es importante destacar las barreras que se han identificado y que dificultan el éxito del proceso emprendedor de la mujer rural. Una de las complicaciones que señalan es la dificultad de mantener una conciliación de la vida laboral y familiar en el caso de tener hijos pequeños a su cargo. Esto es así por la falta de guarderías y por el tiempo que destinan a su profesión. Las mujeres que tienen hijos menores a su cargo son las que mayor importancia le conceden a la posibilidad de compaginar su vida personal y laboral. Este es el caso de la artesana de cerámica y la dueña de una empresa de patrimonio, entre otras.

Estas mujeres señalan que no quieren renunciar a su faceta de madres, por lo que tratan de amoldar su empresa a su vida familiar para poder llevar ambos mundos (personal-profesional), y poder conciliar trabajo y vida familiar, o liberarse de las responsabilidades domésticas y reproductivas (Brunet y Pizzi, 2018). Ante esta problemática tratan de apoyarse en sus parejas y familia, sobre todo en el caso de las empresarias con hijos menores a su cargo.

Las que no tienen hijos también reconocen la dificultad existente en la búsqueda de un equilibrio vital, sobre todo ante la falta de recursos de apoyo externos a la propia familia. Las horas que invierten en sus trabajos son mayores que la jornada media laboral de ocho horas, puesto que terminan por ejercer la labor de empleadores, empleadas, gestoras, administrativas, etc. Son ellas mismas las que desempeñan básicamente todas las figuras profesionales que podríamos encontrar en una organización laboral. En más de una ocasión reconocen el tiempo dedicado a su trabajo y la complicación de compaginar sus vidas con la crianza.

Es difícil, porque mientras no se tienen hijos las cosas son un poco más fáciles a nivel de compaginar la vida familiar y el trabajo, porque el trabajo te exige unas horas de dedicación, que suelen ser muchas, y más cuando eres autónoma, y más cuando estás en un pueblo pequeño. La vida familiar tampoco la puedes descuidar porque si tienes un hijo a ese hijo hay que cuidarle, hay que enseñarle, hay que estar con él. (E5)

No obstante, en los casos en los que no aparecen hijos menores, la familia también sigue teniendo un peso fundamental, como apoyo moral y como dedicación real en sus negocios. Tanto en los comienzos de sus empresas como en su quehacer diario, aparecen figuras como las de los padres y las parejas. Por ello, es común que surja en los discursos el valor que tiene el papel que ellos desempeñan y lo que significa para ellas. En general, indican que han tenido que renunciar a una serie de aspectos (ocio, tiempo, maternidad) para poner en marcha sus proyectos profesionales y para continuar en su andadura. Asimismo, en más de una ocasión señalan que sus trabajos forman parte de un proyecto familiar, más que de una iniciativa únicamente individual. 
Ellos, el apoyo es total, total. Si tenemos que irnos de ferias, pues ellos se quedan con los críos, si vamos a una feria y tienes que montar el stand, ellos vienen a ayudarnos. Si necesitamos material, los llamamos y nos lo traen, o sea, en esto, genial. (E3)

\section{Conclusiones}

Resulta importante señalar que, pese a estudios que han señalado que las mujeres rurales que deciden emprender son mayormente aquellas que heredan negocios familiares o que participan en negocios familiares junto con su pareja (Sampedro y Camarero, 2007), las mujeres de este estudio han emprendido sin necesidad de estos apoyos previos. Es en ellas en las que habría que centrar las políticas públicas, ya que requieren de un mayor apoyo. Estas emprendedoras suponen un importante ejemplo para otras mujeres de la zona y pueden ejercer una influencia como elemento potenciador del territorio y fijar población.

Las complicaciones y los miedos de estas empresarias se localizan fundamentalmente en la puesta en marcha de su negocio. Son coincidentes la fuerza motivadora y la consistencia del criterio que las llevaron a decantarse por sus proyectos.

Entre los elementos transformadores se ha identificado que el medio rural ofrece posibilidades de basar el negocio en las propias inquietudes y gustos personales, ya que no existe tanta competencia como en la ciudad, lo que puede facilitar el asentamiento de población joven y contribuir, así, a frenar la despoblación. Como elemento exclusor cabe destacar la falta de formación emprendedora específica y que ha supuesto la mayor dificultad a la hora de convertir la idea inicial en un recurso rentable. Un ejemplo de política pública que podría reducir la dificultad sería el acompañamiento a través de programas de mentoría o de incubadoras de negocio.

En general, las dificultades con las que se encuentran las mujeres que toman la decisión de constituir su propia empresa en el Somontano son superiores a las que tiene el varón, algo común en el medio rural. Entre ellas destaca también el aspecto económico, la dificultad de acceder a préstamos para poner en marcha el negocio y la dependencia que supone al no tener unos ingresos mensuales garantizados. Por ello, en muchas ocasiones, su actividad empresarial se desarrolla en ramas que requieren menores inversiones iniciales, y que resultan menos fluctuantes al no depender tanto de las variaciones del ciclo económico. Estas mujeres emprendedoras han creado su propio espacio empresarial y se agrupan en la Asociación de Mujeres Emprendedoras del Somontano de Barbastro, insti- 
tución que forma parte de la Asociación de Mujeres Empresarias de la provincia de Huesca, entidad de carácter intersectorial, sin ánimo de lucro e independiente, que agrupa a un amplio colectivo de mujeres empresarias y profesionales, y que se constituye en el año 2007 para la representación y defensa de sus intereses y garantizar la igualdad de hombres y mujeres en el ámbito empresarial.

La ayuda familiar ha sido y es fundamental tanto para desarrollar parte de su actividad como para conciliar su vida personal con la laboral. En todos casos han alcanzado una experiencia en el terreno, aunque aparecen empresarias que tenían una formación educativa previa en el área profesional que desarrollan. No obstante, la proactividad es una actitud destacada en todas ellas, tanto a la hora de continuar con su formación y mejorar sus técnicas como a la hora de tratar de ampliar mercado. Esta inquietud y entusiasmo por reinventarse y mejorarse es una de las habilidades más destacadas de las empresarias rurales.

El autoempleo es, en muchas ocasiones, una forma de solucionar el problema del empleo y constituye una forma de incorporación al mundo laboral de muchas mujeres residentes en el medio rural, dado que pueden compaginar la actividad con las responsabilidades familiares. En la mayoría de las veces, las condiciones en las que se desarrolla el trabajo son deficitarias respecto a la estabilidad, prestaciones sociales, garantía salarial, horarios, etc.

La necesidad de compatibilizar las tareas domésticas con la gestión empresarial o el trabajo autónomo constituye un hándicap para las mujeres residentes en el medio rural que quieren realizar una actividad remunerada fuera del ámbito familiar, estando subordinadas a ello. Es por ello que sería interesante desarrollar también políticas que fomenten la conciliación en el ámbito rural ya que la ausencia de ellas supone una dificultad añadida que enfrentan las mujeres.

Finalmente, es importante señalar que el aumento de empleo autónomo o autoempleo entre las mujeres tiene una doble interpretación: puede ser consecuencia del aumento de los niveles de cualificación femeninos, lo que permite que las mujeres vayan desarrollando cotas más elevadas de autonomía y riesgo profesional, o, por el contrario, es simplemente resultado de la creciente precarización del mercado laboral asalariado, que obliga a muchas de ellas, generalmente con menor nivel de cualificación, a trabajar por cuenta propia en cualquier tipo de actividad, dado que en el medio rural las contrataciones laborales son menores. 


\section{Referencias bibliográficas}

Álvarez, Claudia; Noguera, María y Urbano, David (2017). "Condicionantes del entorno y emprendimiento femenino. Un estudio cuantitativo en España". Economía industrial, 383, 43-52.

Bruin, Anne; Brush, Cándida G. y Welter, Friederike (2006). Introduction to the special issue: towards building cumulative knowledge on women's entrepreneurship. Entrepreneurship: Theory and Practice, 30 (5), 585-593.

Brunet, Ignasi y Pizzi, Alejandro (2018). Discursos de género de las mujeres emprendedoras por oportunidad. El caso español. Revista Austral de Ciencias Sociales, 32, 167-184.

Buttrer, E. Holly (2001). Examining female entrepreneurs' management style: an application of a relational frame. Journal of Business Ethics, 29, 253-269.

Cabezas Hernández, María Teresa (2018). De la mujer rural y el proceso de empoderamiento desde la perspectiva. Almenara: revista extremeña de ciencias sociales, 10, 49-63.

CEDER Somontano (2008). Mujeres empresarias en el Somontano. El saber ayuda a emprender. Barbastro (Huesca): Centro de Desarrollo Integral del Somontano. Disponible en: <https://www.youtube.com/watch?v=aLSbuT7dbI8>.

Consejo Superior de Cámaras de Comercio (2012). Estudio sobre iniciativas empresariales de éxito en el Medio Rural promovida por mujeres. Madrid: Ministerio de Agricultura, Alimentación y Medio Ambiente. Disponible en: <http://www.redm.es/portalredm/images/Estudio\%20de\%20Iniciativas\%20REDM\%20DEFINITIVO\%20completo\%20ver\%202.2.pdf>.

Cruces, Cristina y Palenzuela, Pablo (2006). "Emprendedoras rurales en Andalucía: posibilidades y límites de sus estrategias". Revista española de estudios agrosociales y pesqueros, 211, 239-306.

Díaz Méndez, Cecilia (2005). "Aproximaciones al arraigo y al desarraigo femenino en el medio rural: mujeres jóvenes en busca de una nueva identidad rural." Papers, 75, 63-84.

Escobar Mercado, Modesto, Filardo Llamas, Cristina y Ferrer ArriAzu, Arturo (2005). Autoempleo y actividades empresariales de las mujeres en España. Madrid: Instituto de la Mujer.

Esteban Salvador, María Luisa; Pérez Sanz, Francisco Javier y Gargallo Castel, Ana (2018). "Áreas rurales y cooperativas: iniciativas de mujeres para el desarrollo". REVESCO. Revista de Estudios Cooperativos, 127, 116-138.

Fábregas Martínez, Paloma (2010). Mujeres emprendedoras del Somontano. Avanzando hacia la igualdad. Barbastro: Ayuntamiento de Barbastro y Centro de Desarrollo del Somontano. 
Flecha, Ramón, Vargas, Julio y Dávila, Andrés (2004). "Metodología comunicativa crítica en la investigación en ciencias sociales: la investigación. Workaló". Lan Harremanak. Revista de Relaciones Laborales, (11).

García SAnz, Benjamín (2004a). "La mujer rural en los procesos de desarrollo de los pueblos." Revista del Ministerio de Trabajo y Asuntos sociales, 4, 107-119.

GARCía SANZ, Benjamín (2004b). La mujer rural ante el reto de la modernización de la sociedad rural. Madrid: Instituto de la Mujer.

Heilman, Madeline E. y Chen, Julie J. (2003). "Entrepreneurship as a solution: the allure of self-employment for women and minorities." Human Resources Management Review, 13, 347-365.

Hervás Moreno, Macu, Frutos Mejías, Luisa y Hernández Navarro, María Luz (2016). "Nuevas ruralidades: emprendedoras en turismo rural en el pirineo aragonés". En Ángel Raúl Ruiz Puipón, Manuel Antonio Serrano de la Cruz Santos-Olmo y Julio Plaza Tabasco (coords). Treinta años de Política Agraria Común en España: Agricultura y multifuncionalidad en el contexto de la nueva ruralidad. Ciudad Real: Ciudad Real: Asociación de Geógrafos Españoles (Grupo de Geografía Rural); Editores Óptima.

Jurado Almonte, José Manuel y Pazos García, Francisco J. (2016). "Población y turismo rural en territorios de baja densidad demográfica en España". Boletín de la Asociación de Geógrafos españoles, (71), 247-272.

Jiménez Moreno, Juan J. (2011). Los recursos y resultados empresariales: Una perspectiva de género. Madrid: Instituto de la Mujer.

Langreo, Alicia y Benito, Isabel (2007). Respuesta de las mujeres rurales a los programas operativos y de desarrollo rural del MAPA 2000-2006. Madrid: Ministerio de Agricultura, Pesca y Alimentación.

Ministerio de Medio Ambiente y Medio Rural y Marino (2011). Diagnóstico de la Igualdad de Género en el Medio Rural. Madrid: Secretaria General Técnica.

Nieto Figueras, Cristina (2003). Mujeres empresarias y desarrollo rural. Experiencias en los municipios malagueños (tesis doctoral, manuscrito no publicado). Universidad de Málaga. Departamento de Geografía.

Olson, Su y Walker, Robyn (2004). "Two women and the boys: patterns of identification and differentiation in senior women executives representations of career identity". Women in Management Review, 19 (5/6), 244-251.

Porto Castro, Ana María; Villariño Pérez, Montserrat; Baylina Ferré, Mireia; García Ramón, María Dolors y Salamaña Serra, Isabel (2015). "Formación de las mujeres, empoderamiento e innovación rural". Boletín de la Asociación de Geógrafos Españoles, 68, 385-406. 
Pinilla, Vicente y Sáez, Luis Antonio (2017). La despoblación rural en España: génesis de un problema y políticas innovadoras. Zaragoza: CEDDAR.

Red Europea de mujeres empresarias en el medio rural. [Web]. Disponible en: <http://www.redm.es/portalredm/>.

Sampedro Gallego, Rosario y Camarero Rioja, Luis (2007). "Mujeres empresarias en la España rural. El sujeto pendiente del desarrollo". Revista Internacional de Sociología, 65 (48), 121-146.

Sánchez-Flores, Susana; Royo, Isabel; Lacomba, Joan; Marín, Elvira y Benlloch, Cristina (2014). "Mujeres inmigrantes emprendedoras en el medio rural. Factor para la sostenibilidad económica y social de las áreas rurales de la Comunidad Valenciana". Ager. Revista de Estudios sobre Despoblación y Desarrollo Rural, (16), 69-109.

Santiso Sanz, Raquel (2002). "Hombres y mujeres en el medio rural: el caso del Somontano de Barbastro". Acciones e Investigaciones Sociales, 15, 159-194.

Vega Caro, Luisa y Buzón García, Olga (2016). "Presencia social de mujeres de zonas rurales en las redes sociales". Pixel-Bit. Revista de Medios y Educación, 48, 149-163. 